Gamma-ray Mirrors for Direct Measurement of Spent Nuclear Fuel

M. J. Pivovaroff, K. P. Ziock, M. Fernandez-Perea, M. J. Harrison, R. Soufli

June 17, 2013

INMM 54th Annual Meeting Palm Desert, CA, United States July 14, 2013 through July 18, 2013 
This document was prepared as an account of work sponsored by an agency of the United States government. Neither the United States government nor Lawrence Livermore National Security, LLC, nor any of their employees makes any warranty, expressed or implied, or assumes any legal liability or responsibility for the accuracy, completeness, or usefulness of any information, apparatus, product, or process disclosed, or represents that its use would not infringe privately owned rights. Reference herein to any specific commercial product, process, or service by trade name, trademark, manufacturer, or otherwise does not necessarily constitute or imply its endorsement, recommendation, or favoring by the United States government or Lawrence Livermore National Security, LLC. The views and opinions of authors expressed herein do not necessarily state or reflect those of the United States government or Lawrence Livermore National Security, LLC, and shall not be used for advertising or product endorsement purposes. 


\title{
Gamma-ray Mirrors for Direct Measurement of Spent Nuclear Fuel
}

\author{
Michael J. Pivovaroff ${ }^{1}$, Klaus P. Ziock ${ }^{2}$, Monica Fernandez-Perea ${ }^{1}$, \\ Mark J. Harrison ${ }^{2}$, Regina Soufli ${ }^{1}$ \\ ${ }^{1}$ Lawrence Livermore National Laboratory, Livermore, CA, USA \\ ${ }^{2}$ Oak Ridge National Laboratory, Oak Ridge, TN, USA
}

\begin{abstract}
Direct measurement of the amount of $\mathrm{Pu}$ and $\mathrm{U}$ in spent nuclear fuel represents a challenge for the safeguards community. Ideally, the characteristic gamma-ray emission lines from different isotopes provide an observable suitable for this task. However, these lines are generally lost in the fierce flux of radiation emitted by the fuel. The rates are so high that detector dead times limit measurements to only very small solid angles of the fuel. Only through the use of carefully designed view ports and long dwell times are such measurements possible. Recent advances in multilayer grazing-incidence gamma-ray optics provide one possible means of overcoming this difficulty. With a proper optical and coating design, such optics can serve as a notch filter, passing only narrow regions of the overall spectrum to a fully shielded detector that does not view the spent fuel directly. We report on the design of a mirror system and detector and a number of experimental measurements.
\end{abstract}

\section{Introduction and Motivation}

The non-destructive assay (NDA) of spent fuel is a high priority for the international safeguards community. In the United States, the Department of Energy's (DOE's) National Nuclear Security Administration has started the Next Generation Safeguards Initiative (NGSI), and one of its pillars is technology development. The NGSI spent fuel NDA project has as its primary goal "to enable direct and independent quantification of $\mathrm{Pu}$ mass in spent fuel with an uncertainty of better than 5\%" [1].

Direct measurement of Pu and $\mathrm{U}$ gamma rays is a highly desirable NDA method that could be used in a reprocessing environment, since it does not require inference of the fissile content from measurements of gamma rays from other fission products. However, the high background and intense radiation from directly-viewed spent fuel make direct measurements difficult to implement since the relatively low activities of $U$ and $\mathrm{Pu}$ are masked by the high activity from fission products. To overcome this problem, we propose using grazing-incidence multilayer mirrors to selectively divert hard x-ray and soft gamma rays in the 90-420 keV energy band emitted by $\mathrm{U}$ and $\mathrm{Pu}$ into a high-purity germanium (HPGe) detector shielded from the line-ofsight radiation from spent fuel. This energy range encompasses the elemental-specific $\mathrm{K}$ fluorescence emission lines of $\mathrm{U}$ and $\mathrm{Pu}$, isotopic-specific lines from ${ }^{235} \mathrm{U}$ and ${ }^{239} \mathrm{Pu}$ and lines from other Pu isotopes of interest including ${ }^{238} \mathrm{Pu},{ }^{240} \mathrm{Pu}$ and ${ }^{241} \mathrm{Pu}$. Table 1 lists the primary Kshell fluorescence lines and their relative intensities, and Table 2 lists the brightest nuclear emission lines and their specific activities for individual isotopes.

Robust detection of these lines could provide key information to improve the shipper-receiver difference or input accountability at the start of plutonium reprocessing $[4,5]$. Previous work by several groups [5-8] has shown that it is possible to detect the K-shell fluorescence lines of U and $\mathrm{Pu}$ from spent fuel using germanium detectors. Our goal is to extend this type of 
spectroscopic technique to isotopic-specific lines of $\mathrm{U}$ and $\mathrm{Pu}$ by filtering out unwanted out-ofband emission that can overwhelm the relatively weak lines, when compared to the radiation from other fission products.

Table 1: Primary K-shell fluorescence lines of uranium and plutonium, reprinted from [2].

\begin{tabular}{|c|c|c|c|c|c|}
\hline \multicolumn{2}{|c|}{$\begin{array}{c}\text { Emission line } \\
\text { [designation] }\end{array}$} & \multicolumn{2}{c|}{$\begin{array}{c}\text { Energy } \\
\text { [keV] }\end{array}$} & \multicolumn{2}{c|}{ Relative Intensity } \\
[\%]
\end{tabular}

Table 2: Brightest isotopic emission lines from uranium and plutonium in the $90-420 \mathrm{keV}$ band

\begin{tabular}{|c|c|c|c|c|c|}
\hline Isotope & $\begin{array}{c}\text { Energy } \\
{[\mathrm{keV}]}\end{array}$ & $\begin{array}{c}\text { Specific activity } \\
{[\gamma / \mathrm{g} \times \mathrm{sec}]}\end{array}$ & Isotope & $\begin{array}{c}\text { Energy } \\
{[\mathrm{keV}]}\end{array}$ & $\begin{array}{c}\text { Specific activity } \\
{[\gamma / \mathrm{g} \times \mathrm{sec}]}\end{array}$ \\
\hline${ }^{235} \mathrm{U}$ & 143.8 & $8.76 \times 10^{3}$ & ${ }^{239} \mathrm{Pu}$ & 98.44 & $1.55 \times 10^{5}$ \\
\hline${ }^{235} \mathrm{U}$ & 166.3 & $4.06 \times 10^{3}$ & ${ }^{239} \mathrm{Pu}$ & 111.3 & $3.56 \times 10^{4}$ \\
\hline${ }^{235} \mathrm{U}$ & 185.7 & $4.57 \times 10^{4}$ & ${ }^{239} \mathrm{Pu}$ & 129.3 & $1.44 \times 10^{5}$ \\
\hline${ }^{235} \mathrm{U}$ & 205.3 & $4.01 \times 10^{3}$ & ${ }^{239} \mathrm{Pu}$ & 146.1 & $2.73 \times 10^{4}$ \\
\hline${ }^{238} \mathrm{Pu}$ & 99.85 & $4.49 \times 10^{7}$ & ${ }^{239} \mathrm{Pu}$ & 375.0 & $3.60 \times 10^{4}$ \\
\hline${ }^{238} \mathrm{Pu}$ & 152.7 & $5.90 \times 10^{6}$ & ${ }^{239} \mathrm{Pu}$ & 413.7 & $3.42 \times 10^{4}$ \\
\hline${ }^{241} \mathrm{Pu}$ & 103.7 & $2.26 \times 10^{6}$ & ${ }^{0} \mathrm{Pu}$ & 160.3 & $3.37 \times 10^{4}$ \\
\hline${ }^{241} \mathrm{Pu}$ & 148.6 & $7.15 \times 10^{6}$ & ${ }^{240} \mathrm{Pu}$ & 104.2 & $5.85 \times 10^{5}$ \\
\hline${ }^{241} \mathrm{Pu}$ & 208.0 & $2.04 \times 10^{7}$ & & & \\
\hline
\end{tabular}

In Section 2, we discuss the basic properties of the reflective multilayer mirrors and recent developments in their fabrication that we have leveraged to produce mirrors at the DOE's Lawrence Livermore National Laboratory (LLNL) appropriate for nuclear safeguards. We then summarize the results of an extensive measurement campaign used to validate that the performance of the multilayer elements had the desired characteristics.

In Section 3, we discuss a proof-of-concept experiment conducted at the DOE's Oak Ridge National Laboratory (ORNL) using sealed radioactive sources to show the efficacy of multilayer mirrors as a pass-band filter for reflecting specific emission lines into a HPGe detector. Finally, in Section 4 we briefly describe our plans for the future, which include using gamma-ray mirrors to measure spent nuclear at the Irradiated Fuels Examination Laboratory (IFEL) at ORNL.

\section{Reflective $x$-ray optics}

\subsection{Background}

It was Arthur Compton [9] who first realized that x-rays incident on a surface at a shallow or grazing angle (of order a few degrees or less) can undergo total external reflection from the surface of a material because the index of refraction $n$ is less than unity at x-ray wavelengths. 
The index of refraction is commonly written as $n=1-\delta-i \beta$, where the real part, $\delta$, is the refractive index decrement and the imaginary part, $\beta$, is the absorption index and proportional to the atomic photoabsorption cross-section. The transition between the total external reflection and absorption happens at the critical angle $\theta_{c}$, where $\theta_{c}=\sqrt{2 \delta}$ and depends strongly on the material and the photon energy $E$.

Reflective x-ray optics have been developed primarily for two scientific applications: x-ray light sources (like synchrotrons and free electron lasers) and astronomy and astrophysics. At x-ray synchrotrons or free electron lasers, the optics comprise elements in a beamline and serve to condition, spectrally filter or focus the x-rays as they propagate to an end-station for use in a scientific investigation. Examples of this include the primary mirror systems used at the Linac Coherent Light Source (LCLS), the x-ray free electron laser operating at the DOE's SLAC National Accelerator Laboratory [10].

Focusing $x$-ray optics allow true imaging and the first practical design was proposed by Kirkpatrick and Baez in 1948 [11]. Now called Kirkpatrick-Baez or KB optics, these optical designs require two mirror elements - the first focusing in one dimension, the second focusing perpendicular to the first - and are widely used at light sources or in other applications where the source flux is high. In 1952, Wolter [12] proposed a new focusing design requiring pairs of mirrors built from surfaces of revolution of conic sections (e.g., hyperboloids and ellipsoids) that function together to achieve excellent focusing properties across a wide field of view (FOV). Figure 1 illustrates the basic imaging concepts of a KB and Wolter x-ray optic.
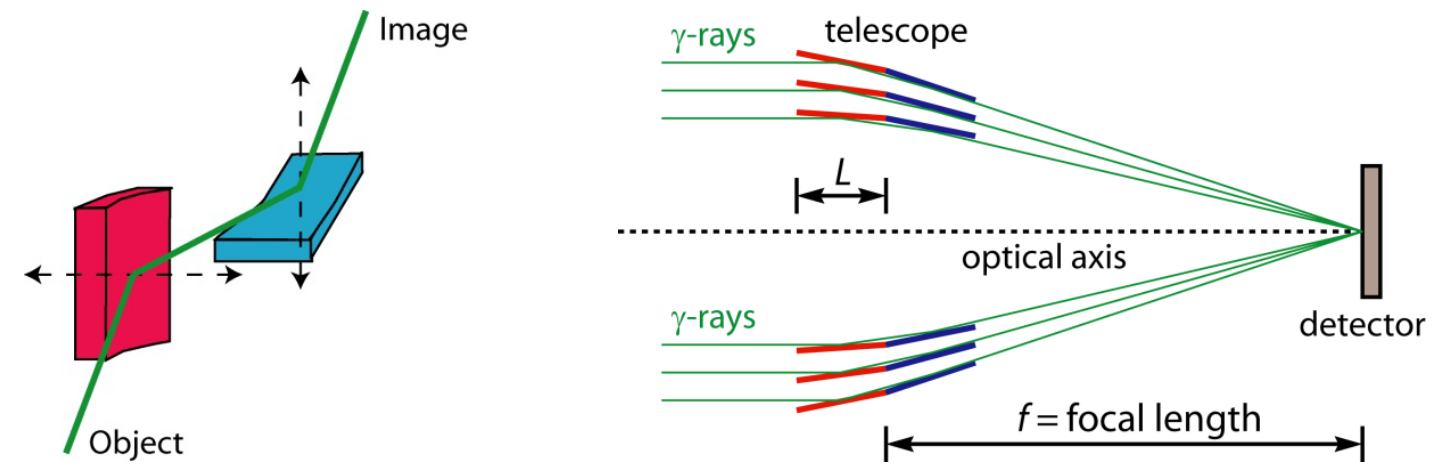

Figure 1. [Left:] Schematic of Kirkpatrick-Baez (KB) optic. The photons are first focused in the horizontal direction by the red mirror, then focused in the vertical direction by the blue mirror. [Right:] Schematic cross-section through a Wolter optic. Three nested shells are shown. The photons are first reflected from parabolic mirrors (red), then hyperbolic mirrors (blue).

Although Wolter had envisioned using such optics for biological studies, the first successful implementation of his designs was employed for an x-ray telescope in the late 1960s [13]. Since then, the high-energy astrophysics community has spent billions of dollars building and refining reflective x-ray optics for many satellite-based observatories. An important realization of the community was that sets of co-focal mirrors could be placed inside one another, resulting in a nested optical system to increase the collection efficiency by orders of magnitude above that of a single pair of mirrors. During the last two decades, space-based x-ray satellites like ASCA, 
$X M M$-Newton and NuSTAR have used nested telescopes consisting of several tens to more than one hundred nested layers to achieve large collecting areas [14,15,16].

Even with practical optics designs, until recently, the useful upper limit to the energy of such optics was limited to $E \approx 10 \mathrm{keV}$ because of the extremely small graze angles required for use at higher energies. To extend the energy band, several groups proposed using multilayer coatings in conjunction with Wolter-like optical designs $[17,18]$. Just as multilayers can affect the transmission or reflection of visible wavelength light, alternating layers of material with a differing $n$ can affect the reflection of x-rays. Coatings made of alternating layers of low- and high-density materials cause the radiation to constructively interfere and obey Bragg's law: $m \lambda=2 d \sin \theta$, where $m$ is an integer representing the order of the reflection, $\lambda$ is the wavelength of the photon, $d$ is the period of the multilayer and $\theta$ is the graze angle. Figure 2 illustrates the two broad classes of multilayer recipes: constant-d spacing coatings, designed to have the same period throughout the coating to work for a specific angle and photon energy $(E \propto 1 / \lambda)$ and graded-d spacing (depth-graded) coatings, designed to have a range of periods throughout the coating and to work over a range of energies and angles. Whatever the design, fabricating coatings with small period thickness $d$ makes it possible to work at larger graze angles, for a given energy, or at higher energies, for a given angle.
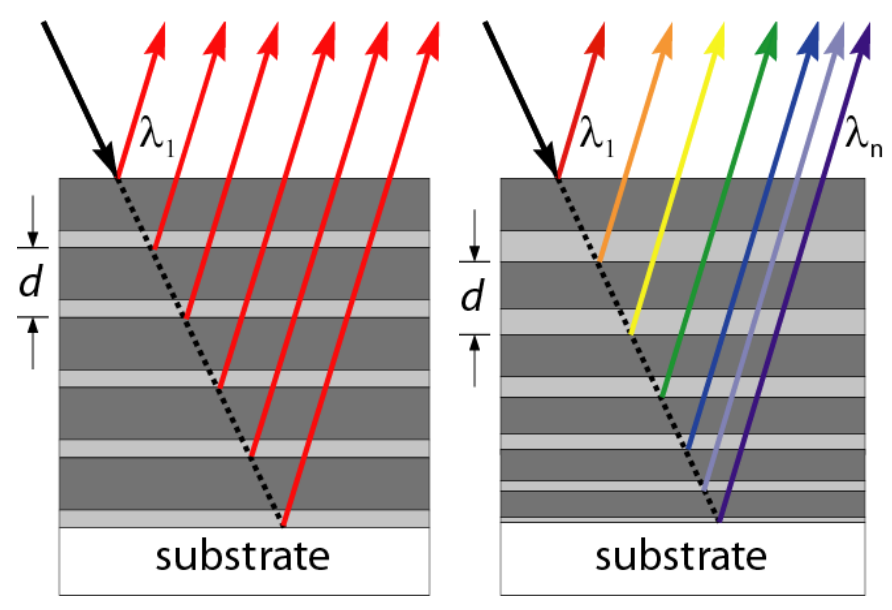

Figure 2. Illustration of multilayer designs. [Left:] In a constant-d spacing design, the thickness $d$ of the alternating layers of high- and low-density materials are the same throughout the coating. This maximizes reflectivity for a unique combination of wavelength $(\lambda \propto 1 / E)$ and graze angle $\theta$. [Right:] In a graded-d spacing (depth-graded) design, the thickness $d$ of the bi-layer is varied, allowing Bragg's law to be satisfied for a range of energies and angles.

The highest energy reflective $\mathrm{x}$-ray multilayer optics currently used for an operational application are the two telescopes flying onboard NuSTAR, a NASA satellite launched in June 2012 [16]. Each telescope consists of more than 2000 individual x-rays mirrors, consisting of a curved glass substrate on which is deposited either $\mathrm{Pt} / \mathrm{C}$ or W/Si depth-graded multilayer coatings designed to provide high collection efficiency up to $79 \mathrm{keV}$ [19]. The success of NUSTAR proves complex, reflective multilayer optics can operate at shallow graze angles of $1.3-4.7 \mathrm{mrad}\left(0.074-0.27^{\circ}\right)$. In order to use multilayers for non-destructive assay of spent fuel, we had to: (1) prove that multilayers can work well at energies $E=100-400 \mathrm{keV}$ in order to measure the lines listed in Tables 1 and 2; (2) prove that we could regularly fabricate multilayers with periods of $d=1-2 \mathrm{~nm}$ so that we could operate the gamma-ray mirrors at graze angles of a few mrad. 


\subsection{Development of gamma-ray mirrors}

During the last four years, our team of scientists and engineers from LLNL and ORNL has systematically developed and tested the necessary multilayer coatings to work efficiently in the soft gamma-ray band of 100-400 keV. Details of this work have been published in 2013 in two refereed papers $[20,21]$ and are briefly summarized below.

$\mathrm{WC} / \mathrm{SiC}$ was determined to be an excellent material system to use for gamma-ray applications. It can be engineered to simultaneously have low-stress and form good interfaces, (i.e. with minimal intermixing of WC and SiC. These properties are required to achieve high reflectivity in the soft gamma-ray band. After optimization of the deposition process, we found that it is possible to deposit multilayer coatings consisting of several hundred bi-layer pairs with periods of $d=1-2$ $\mathrm{nm}$. To understand how these candidate mirrors would perform at high energies, we fabricated several reference standard mirrors for detailed at-wavelength characterization.

The mirror substrates are highly polished flat quartz of size $150 \mathrm{~mm} \times 150 \mathrm{~mm} \times 6.4 \mathrm{~mm}$. The WC/SiC multilayers have $d=1.0,1.21 .5$ and $2.0 \mathrm{~nm}$ and have a range of $N=300-500$ bi-layer pairs. After coating, the mirrors were measured with a full-aperture interferometer to determine their flatness; mirrors had a figure error of $\sim 1 \mu \mathrm{rad}(0.2 \mathrm{arcsec})$ in the central $40 \times 40 \mathrm{~mm}^{2}$ region and of $3 \mu \mathrm{rad}(0.6 \mathrm{arcsec})$ across the entire face of the mirror.

These reference mirrors were systematically studied at $8 \mathrm{keV}$ at DTU-Space (Copenhagen, Denmark), at $62 \mathrm{keV}$ and $186 \mathrm{keV}$ at the National Synchrotron Light Source (NSLS, located at DOE's Brookhaven National Laboratory) and at $378 \mathrm{keV}$ and $384 \mathrm{keV}$ at the European Synchrotron Radiation Facility (ESRF, located in Grenoble, France). We found that the mirrors with 1.5 and $2.0 \mathrm{~nm}$ periods have excellent high-energy performance and that we can achieve reflectivity of greater than $50 \%$ at $384 \mathrm{keV}$ for our $d=1.5 \mathrm{~nm}$ multilayer mirror. Figure 3 (left) shows $378 \mathrm{keV}$ results from several mirrors studied at ESRF.
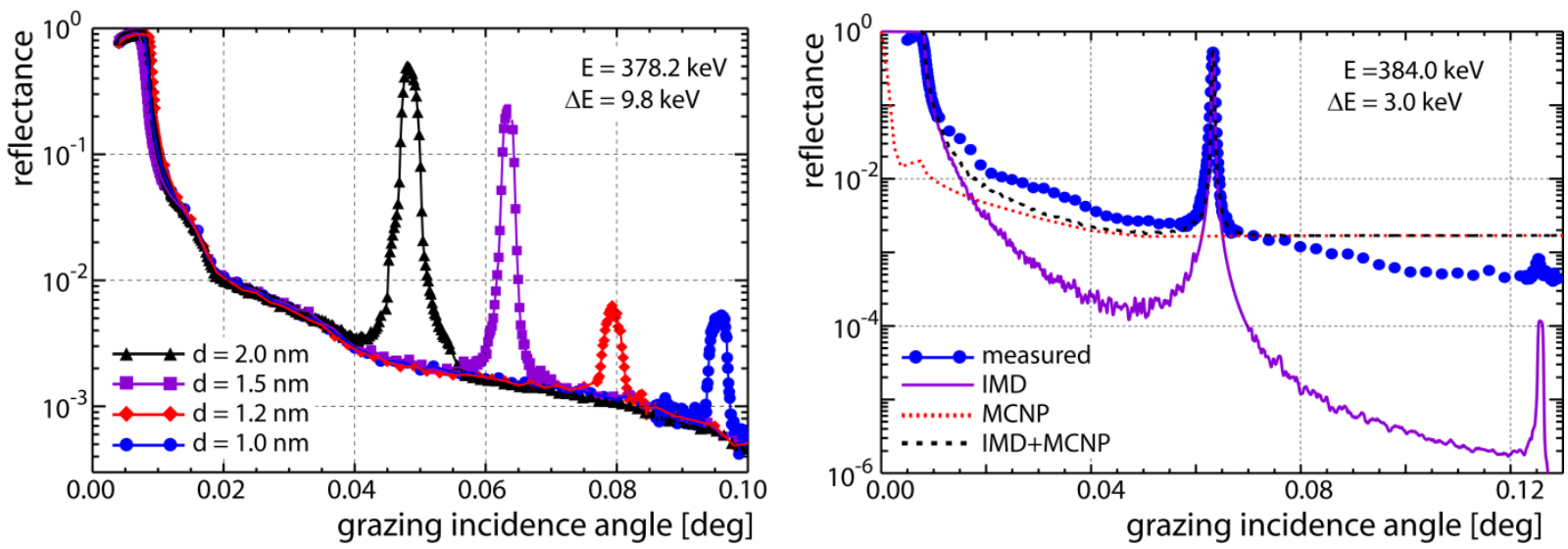

Figure 3. [Left] Multilayer reflectivity versus d-spacing. High reflectivity is possible with multilayer periods (d) of $1.5 \mathrm{~nm}$ and $2.0 \mathrm{~nm}$. Reflectivity is significantly lowered when the period is reduced to 1.2 $\mathrm{nm}$ and $1.0 \mathrm{~nm}$. [Right] Multilayer reflectivity versus angle. The measured data (blue points) is welldescribed by a model (black curve) that includes constructive interference (purple curve) and incoherent scatter (red curve). 
An important outcome of the measurement campaign is that we can accurately predict the gamma-ray reflectance of the mirrors using models derived from the 8- and 62-keV data using the IMD software developed by David Windt [22]. This has two important implications. First, it means calibration can, in part, be performed at lower-energy, non-synchrotron facilities, which are much more plentiful and easier to use. Second, it means performance can be predicted using extrapolation of known optical constants and that problems with the use of these constants reported by others [23-25] above $100 \mathrm{ke} \mathrm{V}$ are not an issue for designing a full optical system at these energies. Finally, we note that while the amplitude and location of the Bragg peaks can be predicted using a wave-optics formalism, to accurate predict the reflectance results across the entire angular range, one must also account for inelastic scattering (and the particle-like nature of the photons) in the gamma-ray mirror and the beamline using a Monte Carlo radiation transport code like MCNP6 [26]. Figure 3 (right) shows the agreement between the composite model and the data at $384 \mathrm{keV}$ for the $d=1.5 \mathrm{~nm} \mathrm{WC/SiC}$ multilayer mirror.

\section{Initial demonstration of a multilayer pass-band filter}

Initial proof-of-concept was achieved using a tightly collimated ${ }^{241} \mathrm{Am}$ source, one of the $d=1.5$ nm reference mirrors discussed above, and a high-purity germanium (HPGe) gamma-ray spectrometer aligned on an optics table. Figure 4 illustrates the basic setup utilized to perform the measurements, and Table 3 describes various elements of the experiment.

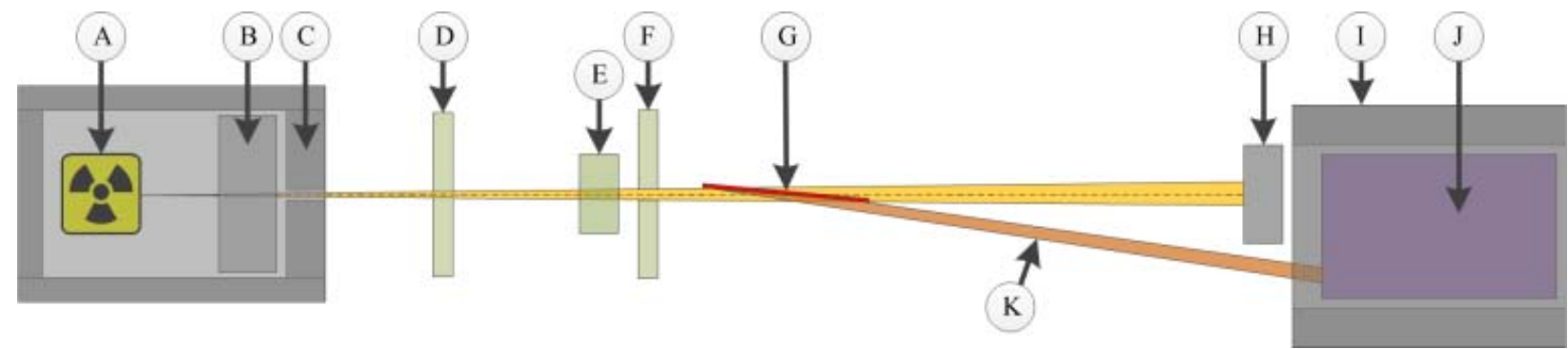

Figure 4. Illustration of the experimental setup. See Table 3 for a detailed description of each of the elements indicated by letters $\mathrm{A}-\mathrm{J}$.

Table 3. Details on key elements of the experiment illustrated in Figure 4.

\begin{tabular}{|c|l|l|}
\hline Element & \multicolumn{1}{|c|}{ Name } & \multicolumn{1}{c|}{ Description } \\
\hline A & Sources & $3.294 \mathrm{mCi}^{241} \mathrm{Am}, 1 \mathrm{mCi}{ }^{133} \mathrm{Ba}$ \\
\hline $\mathrm{B}$ & Coarse vertical collimator & Milled Pb bricks \\
\hline $\mathrm{C}$ & Coarse horizontal collimator & Milled Pb bricks \\
\hline $\mathrm{D}$ & $1^{\text {st }}$ fine horizontal collimator & Two W-alloy blocks mounted to manual linear stages \\
\hline $\mathrm{E}$ & Fine vertical collimator & Two W-alloy blocks mounted and spaced manually \\
\hline $\mathrm{F}$ & $2^{\text {nd }}$ fine horizontal collimator & Two W-alloy blocks mounted to manual linear stages \\
\hline $\mathrm{G}$ & Mirror & $\begin{array}{l}\text { Multi-layer mirror mounted to rotary and linear stages } \\
\text { WC/SiC, } N=300, d=1.5 \text { nm }\end{array}$ \\
\hline $\mathrm{H}$ & & Pb brick \\
\hline $\mathrm{I}$ & Scatter shield & 2 inch thick Pb cylinder \\
\hline $\mathrm{J}$ & HPGe detector & Ortec GEM-X8550P-S \\
\hline $\mathrm{K}$ & Reflected beam & \\
\hline
\end{tabular}


Element (A) indicates the radioactive source(s). Two sources were utilized in performing the measurements described later. The primary source was a ${ }^{241} \mathrm{Am}$ source $(3.294 \mathrm{mCi}$ activity at time of experiment). The 'background' source from which the $59.5 \mathrm{keV}$ line of ${ }^{241} \mathrm{Am}$ was discriminated was a $1 \mathrm{mCi}{ }^{133} \mathrm{Ba}$ source that was used only when indicated. (B) is the first horizontal collimator set to have a gap width of $1.2 \mathrm{~mm}$ using $\mathrm{Pb}$ bricks that had been milled flat. (C) was the first vertical collimator with a gap width of $3.4 \mathrm{~mm}$, again using milled-flat $\mathrm{Pb}$ bricks. (D) and (F) were each horizontal collimators constructed of two W blocks each mounted to a manual horizontal stage so that each collimator pair could be adjusted to the desired beam width and location. (E) was a finely tuned horizontal collimator that utilized milled W blocks. (G) was the multi-layer mirror mounted to an Al holder which was attached to a motorized rotary stage and a motorized horizontal stage. The rotary stage allowed for the adjustment of the angle that the beam was incident upon the mirror, and the design included a linear stage for placing and removing the mirror from the beam path. A large $\mathrm{Pb}$ tube shield (I) was placed around the HPGe detector (J) to suppress counts due to room scatter. A beam stop $(\mathrm{H})$ was used to block those photons passing straight through the multi-layer mirror. Photons reflected off the mirror (K) were set to miss the beam stop $(\mathrm{H})$ but be incident upon the HPGe (J).

The set up and alignment of the experimental apparatus required several steps. First, a diffuse light source was placed at $(A)$ while elements $(B)$ through $(\mathrm{H})$ were removed from the beam incident upon the HPGe (J). Beginning with (B) and moving toward (H), each element except (G) was brought into position to define the desired beam spot on the face of the detector. Next, the diffuse light source at (A) was removed and placed at the location at which the beam was incident upon the detector. This allowed a beam of visible light to pass back through the collimators. The location at which this beam of light struck the back wall of the laboratory was marked and the light source at (J) was removed. Next, a laser was mounted at (A) and the mirror was brought into the beam. The mirror was adjusted to be perpendicular to the beam until the laser beam aligned with the previously marked point on the back wall. This step provided an angular reference point for mirror calibration. The mirror was then turned to the desired angle and its horizontal alignment adjusted until the laser beam struck the center of the mirror. At this point, the laser beam traced out path $(\mathrm{K})$ and the mirror's angle was finely calibrated. As a last step, the mirror was moved back a known amount and the diffuse light source placed at $(\mathrm{H})$ again. The laser pointer was removed at (A) and the source or sources were placed at (A) and aligned to the light spot defined by the collimators and the diffuse light source at $(\mathrm{H})$. This final calibration ensured the source(s) at (A) were properly aligned to the beam. Lastly, the mirror was replaced to its calibrated position.

The first measurement determined how well the beam stop and collimation system reduced noise. In this measurement, a 'background ${ }^{133} \mathrm{Ba}$ source was placed in conjunction with the ${ }^{241} \mathrm{Am}$ source at (A), the mirror was retracted from the beam and spectra were collected with and without the beam stop $(\mathrm{H})$ in place. The spectra are provided in Figure 5 (left) showing a reduction in signal magnitude by approximately two orders of magnitude at $59.5 \mathrm{keV}$. This confirmed that the $59.5 \mathrm{keV}$ emission of ${ }^{241} \mathrm{Am}$ was effectively blocked by the beam stop. 
Next, the ability of the mirror to reflect $59.5 \mathrm{keV}$ gamma rays was measured by placing only the ${ }^{241} \mathrm{Am}$ source at (A) and placing the mirror in position. Here, the angle of the mirror was varied and differential energy spectra collected for each position. Figure 5 (right) provides an example of the spectra collected with and without the mirror in position. Most notably the $59.5 \mathrm{keV}$ net peak intensity increased significantly from 250 net counts per hour to 1450 net counts per hour, a factor of $5.8 \times$ increase. This result clearly confirmed the efficacy of the multilayer mirror in reflecting $59.5 \mathrm{keV}$ gamma rays.
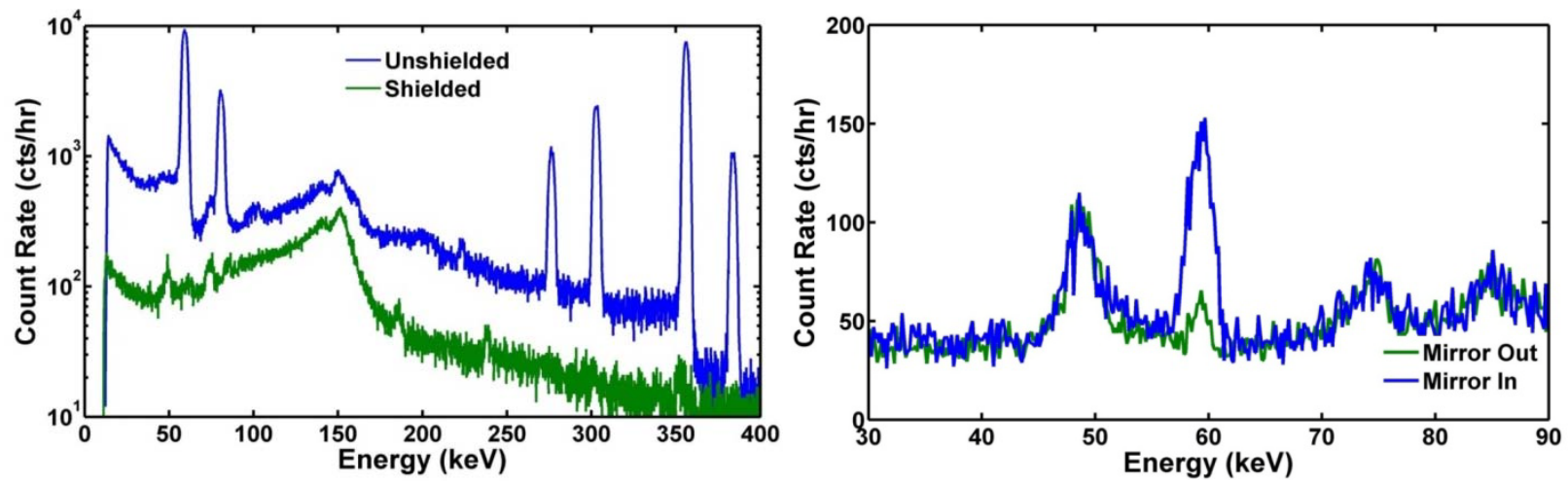

Figure 5. [Left] Measured spectra of the ${ }^{241} \mathrm{Am}$ and ${ }^{133} \mathrm{Ba}$ sources without and without the beam stop in place at $(\mathrm{H})$. [Right] Energy spectra with and without the multi-layer mirror in place with only the ${ }^{241} \mathrm{Am}$ source present.

Finally, the ${ }^{133} \mathrm{Ba}$ source was added to the ${ }^{241} \mathrm{Am}$ source at (A) and the net counts in the $59.5 \mathrm{keV}$ peak were recorded for each mirror angle. Figure 6 (left) is a plot of the net counts in the 59.5 $\mathrm{keV}$ photopeak as a function of mirror angle. It can be seen that the peak count rate occurred at an angle of $0.275^{\circ}$, lower than expected from simulation. This however is most likely a result of a slight $\left(0.2^{\circ}\right)$ misalignment of the mirror. Furthermore, the small $\sim 0.14^{\circ}$ FWHM of the curve in Figure 6 (left) and its asymmetry indicate the mirror surface was not exactly aligned to the axis of rotation.
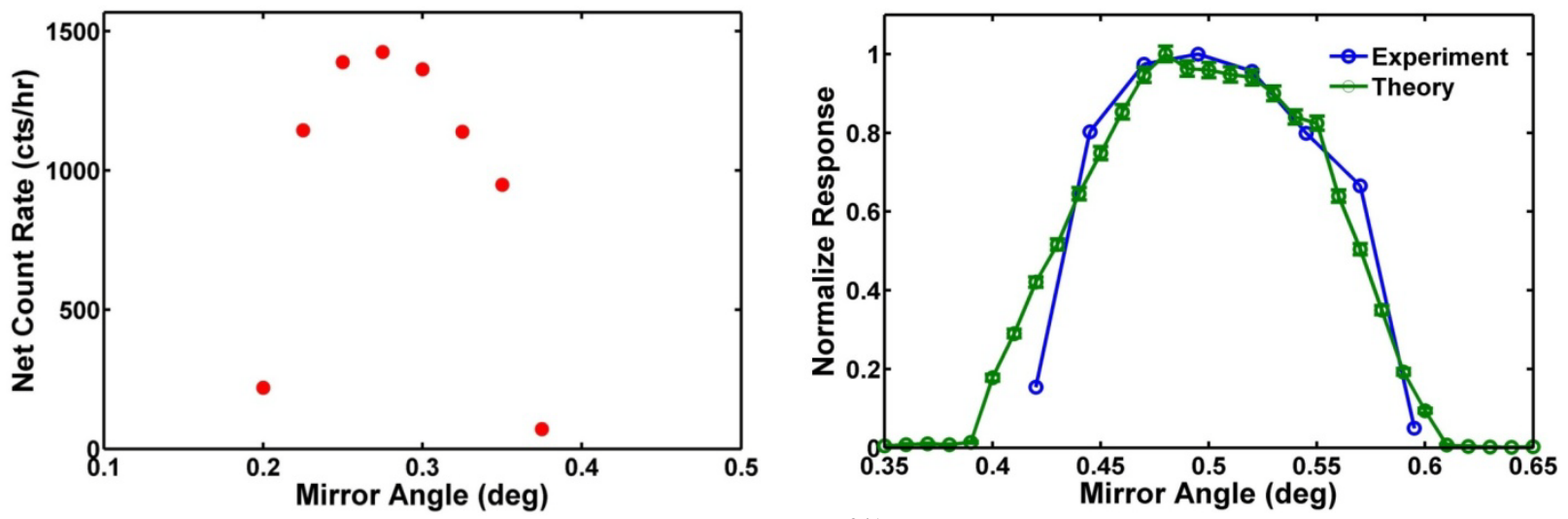

Figure 6. [Left] Net counts recorded in the $59.5 \mathrm{keV}\left({ }^{241} \mathrm{Am}\right)$ photopeak as a function of mirror angle. [Right] Comparison between experimentally collected data and theoretical results assuming a $0.2^{\circ}$ misalignment of the mirror angle and a $1.8 \mathrm{~mm}$ offset between the mirror surface and its axis of rotation. 
In fact, comparing the experimentally measured rocking curve in Figure 6 (left) and accounting for its angular shift, good agreement can be found with theory when it is assumed that the mirror surface is offset from the axis of rotation by $1.8 \mathrm{~mm}$. Figure 6 (right) shows this agreement in peak width and shape.

\section{Plans for the future: instrumentation to study spent fuel}

We have begun construction of a simple multilayer mirror assembly to study spent fuel at the Irradiated Fuels Examination Laboratory (IFEL) at ORNL. The mirror assembly consists of flat silicon substrates deposited with constant-period WC/SiC multilayers with $d=1.5 \mathrm{~nm}$ that are aligned parallel to one another. A custom collimator, with an angular adjustment mechanism, allows the mirror assembly a direct view of spent fuel contained in a hot cell. A HPGe detector with two-dimensional readout will record the spectra of the reflected emission. Measurements at the IFEL are scheduled for the summer of 2013.

\section{Acknowledgements}

This work was performed under the auspices of the U.S. Department of Energy by Lawrence Livermore National Laboratory under Contract DE-AC52-07NA27344 and by Oak Ridge National Laboratory, managed by UT Battelle, under contract DE-AC05-000R22725. The project is funded by the U.S. Department of Energy Office of Proliferation Detection, NA-221, through grants LL10-GammaMirror-PD03 and OR10-GammaMirror-PD03.

\section{References}

1. M. Humphrey. "Nondestructive Assay of Spent Fuel for International Safeguards," 16-Jan2013, accessed 20-May-2013 http://ramp.energy.gov/docs/education/Q17.pdf

2. D. Reilly, N Ensslin, S. Smith Jr., and S. Kreiner. Passive Nondestructive Assay of Nuclear Materials (1991); Passive Nondestructive Assay of Nuclear Materials, Addendum, ed. D. Reilly (2007).

3. D. Clark. “U235: A Gamma Ray Analysis Code for Uranium Isotopic Determination”, UCRL-ID-125727 (December 1996).

4. T. Hayakawa et al. "Nondestructive assay of plutonium and minor actinide in spent fuel using nuclear resonance fluorescence with laser Compton scattering $\gamma$-rays," Nuclear Instruments and Methods A, 621:695 (2010).

5. A. S. Stafford. "Spent Nuclear Fuel Self-Induced XRF to Predict Pu to U Content”, M.S. Thesis (August 2010).

6. A. V. Bushuev et al. "A Nondestructive Method of Determining the Pu/U Ratio in Fast Reactor Fuel Elements, Based on X-ray Spectrometry,” Atomic Energy 53:5 (1982)

7. C. Rudy, P. Staples, K Serednuik and I. Yakovlev. "Determination of Pu in Spent Fuel Assemblies by X-ray Fluorescence," Proceedings of $46^{\text {th }}$ Annual Meeting of the INMM, (2005).

8. W. S. Charlton et al. "The Use of Self-Induced XRF to Quantify the Pu Content in PWR Spent Nuclear Fuel," Proceedings of the $31^{\text {st }}$ Annual Meeting of ESARDA (2009).

9. A. H. Compton. "The Total Reflection of X-rays,” Philosophy Magazine, 45:1121 (1923).

10. M. J. Pivovaroff et al. "Soft X-ray mirrors for the Linac Coherent Light Source," Proceedings of the SPIE, 6705:67050O (2007). 
11. P. Kirkpatrick and A. V. Baez "Formation of Optical Images by X-Rays," Journal of the Optical Society of America, 38:766 (1965).

12. H. Wolter. "Spiegelsysteme streifenden Einfalls als abbildende Optiken fur Rontgenstrahlen,“ Annalen der Physik 10:94 (1952).

13. R. Giacconi, NF Harmon, RF Lacey and A Szilagyi. "Aplanatic telescope for soft x rays," Journal of the Optical Society of America, 55:345 (1965).

14. Y. Tanaka, H. Inoue, and S. S. Holt. "The X-ray astronomy satellite ASCA,” Proceedings of the Astronomical Society of the Pacific 48:L33 (1994).

15. F. Jansen et al. "XMM-Newton observatory. I. The spacecraft and operations," Astronomy and Astrophysics, 365:L1 (2001)

16. F. A. Harrison et al. "The Nuclear Spectroscopic Telescope Array (NuSTAR) High-energy X-ray Mission,” Astrophysical Journal, 770:130 (2013).

17. A. B. C. Walker et al. "Astronomical observations with normal incidence multilayer optics: recent results and future prospects,” Physica Scripta, 41:1053 (1990).

18. F. E. Christensen et al. "A graded d-spacing multilayer telescope for high energy X-ray astronomy," Proceedings of the SPIE, 1546:160 (1992).

19. C. J. Hailey et al. "The Nuclear Spectroscopic Telescope Array (NuSTAR): Optics Overview and Current Status," Proceedings of the SPIE, 7732:77320T (2010).

20. M. Fernandez-Perea et al. "Ultra-short-period WC/SiC multilayer coatings for x-ray applications," Nuclear Instruments and Methods A, 710:114 (2013).

21. M. Fernandez-Perea et al. "Physics of reflective optics for the soft gamma-ray photon energy range,” Physical Review Letters, accepted (2013).

22. D. L. Windt. "IMD - Software for modeling the optical properties of multilayer films," Computers in Physics, 12:360 (1998).

23. D. L. Windt et al. "W/SiC X-ray multilayers optimized for use above $100 \mathrm{keV}$," Applied Optics, 42:2415 (2003).

24. C. P. Jensen, S Romaine, R Bruni, FE Christensen, Z Zhong. "Optical constants in the hard $\mathrm{X}$-ray/Soft gamma-ray range of selected materials for multilayer reflectors," Proceedings of the SPIE, 6688:66880V (2007).

25. C. P. Jensen et al. "Optical constants in the hard X-ray/Soft gamma-ray range," Proceedings of the SPIE,7011:70111K (2008).

26. T. Goorley et al. “Initial MCNP6 Release Overview,” Nuclear Technology, 180:298 (2012). 